\title{
One-staged combined decompression for the patients with cervico-thoracic tandem spinal stenosis
}

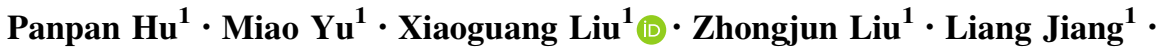 \\ Zhongqiang Chen ${ }^{1}$
}

Received: 13 September 2015/Revised: 22 February 2016/ Accepted: 23 February 2016/Published online: 7 March 2016

(c) The Author(s) 2016. This article is published with open access at Springerlink.com

\begin{abstract}
Purpose To report the techniques and safety of onestaged combined decompression for the patients with tandem spinal stenosis (TSS) at cervical and thoracic spine.

Methods Sixteen TSS subjects, who received combined decompression from Aug 2005 to Feb 2012, were reviewed. The essentials of our surgical strategy included: choosing patients with TSS from cervical to upper or middle thoracic spine, using one single posterior incision, simplifying surgical maneuvers and performing circumferential decompression for thoracic compression if it was indicated. The Japanese Orthopedic Association (JOA) scale for cervical myelopathy was employed to evaluate the neurological status, and Hirabayashi's system to assess neurological recovery rate.

Results The average operation duration, blood loss and postoperative hospitalization were $242.8 \pm 89.9 \mathrm{~min}$, $1581.3 \pm 1237.2 \mathrm{ml}$ and $11.9 \pm 7.5$ days, respectively. Six subjects $(37.5 \%)$ suffered instant neurological deterioration. Other complications included cerebrospinal fluid leakage (10 subjects, $62.5 \%$ ), new radiculopathy (two subjects), urinary infection, lung infection and pulmonary thromboembolism. Four subjects received extra-thoracic
\end{abstract}

P. Hu and M. Yu are equal contributors and share the first authorship.

Electronic supplementary material The online version of this article (doi:10.1007/s00586-016-4497-1) contains supplementary material, which is available to authorized users.

\section{Xiaoguang Liu}

puth_1xg@163.com

1 Department of Orthopedics, Peking University Third Hospital, No. 49 Huayuanbei Rd, Haidian District, Beijing 100191, China decompression due to the remaining anterior compression in one subject and new emerging compression in other three subjects. Eventually, mean JOA score was elevated from $9.8 \pm 2.1$ to $13.7 \pm 2.7$ after this procedure, and the neurological recovery of seven subjects was rated as excellent, four as good, two as fair, three as unchanged or deteriorated. The overall recovery rate was $53.7 \%$.

Conclusion Combined cervico-thoracic decompression could provide fair neurological outcomes for patients with cervico-thoracic TSS, but it was complicated with high rate of undesirable postoperative events. So, more efforts should be done against its eventful postoperative course before its wide application.

Keywords Tandem spinal stenosis - Cervical spine . Thoracic spine $\cdot$ One-staged decompression · Surgical outcomes

\section{Introduction}

Spinal stenosis is an age-associated disease and can sometimes involve more than one spinal segment, a situation which is termed as tandem spinal stenosis (TSS) [1, 2]. Occasionally, symptomatic cervico-lumbar and cervicothoracic TSS were reported in previous publications, within which the results of different surgical approaches were described [1, 3-6]. Generally, cervico-lumbar TSS is related to the spondylotic proliferation and disc herniation, while cervico-thoracic TSS is more likely to result from ectopic ossification and bulking of ligaments inside the spinal canal, namely ossification of posterior longitudinal ligament (OPLL) and ligamentum flavum (OLF) [4, 5]. Similar to cervico-lumbar TSS, two surgical strategies are 
available for the cervico-thoracic TSS, which are onestaged and two-staged decompressions. One-staged combined decompression seems more attractive due to the potential benefits of single hospitalization and single anesthetic procedure. Chen et al. [5] did the pioneering research for one-staged surgery in 15 patients with cervicothoracic TSS, and regarded it as an aggressive surgical strategy. They have emphasized on the preoperative communication with patients on the impact of perioperative complications and postoperative consequences on its outcome. The surgical maneuvers and/or approaches were diverse in the study by Chen et al., for example, both anterior and posterior approaches for cervical spine were used. In this study, we report the clinical outcomes of onestaged combined decompression via a single posterior incision, which could be regarded as an attempt to simplify the procedure.

\section{Materials and methods}

\section{Materials}

Patients with cervico-thoracic TSS were reviewed, from Aug 2005 to Feb 2012. The inclusion criteria for this study were as follows: (1) patients who presented with symptoms or signs of neurologic deficits in both upper and lower extremities; (2) preoperative magnetic resonance imaging (MRI) scans demonstrated spinal stenosis and abnormal signals within the spinal cord in both cervical and thoracic spine; (3) complete records of demographic and operative information were available; (4) regular follow-up for more than 3 years has been done.

Finally, a cohort of 16 subjects were recruited (detailed information for each subject are given in Online Resource 1), and all took cervico-thoracic combined decompression by senior surgeons (X. Liu and Z. Liu) in our hospital, via a single posterior incision. All the recruited subjects had walking problems to different extents, 12 subjects reported numbness or other sensory impairment in the trunk and/or the lower extremities, four subjects complained of sting feeling, numbness and/or weakness in the upper extremities, and nine subjects suffered sphincter dysfunction, among whom two subjects were admitted with urinary catheterization. Physical examinations demonstrated signs of neurological deficits in both upper and lower limbs among all 16 subjects. Imaging work-ups showed that the tandem stenosis ran from cervical spine to upper or middle thoracic spine in all recruited subjects and particularly, thoracic OPLL was found in 13 subjects, among whom 10 subjects presented with cervical OPLL.

\section{Methods}

\section{Surgical procedures}

The patients lay prone and the heads were fixed by Mayfield holder. All procedures were performed under general anesthesia. The decompression levels were determined according to patient's neurological symptoms, physical examination, as well as imaging findings. Generally, the rostral range of the decompression was the level or one level above the upper end of compressive cervical lesion and the caudal range of the decompression was usually one level below the lower end of compressive thoracic lesion. Cervical decompression was accomplished via left opendoor laminoplasty (ODL) in 14 subjects, with the open space at $1.5-2.0 \mathrm{~cm}$, and laminectomy with lateral-mass fusion (LLF) in two subjects (Table 1; Fig. 1).

For thoracic decompression, standard laminectomy was initially undertaken with or without pedicle screw-assisted fusion (Table 1). Then the sufficiency of decompression was assessed by a combination of different modalities, including the observation of refilling and pulsating of the dural sac, feeling its tension, alterations of electrophysiological monitoring, intraoperative ultrasound to detect backward shift of the spinal cord and the space between anterior compression and the dural sac, microbubbles (sulphur hexafluoride microbubbles) contrast-enhanced ultrasound to evaluate blood reperfusion to the spinal cord. Noticeably, intraoperative ultrasound was used only for the last five subjects (Fig. 1). If there was suspicion or evidence for remaining ventral compression, circumferential decompression (CD) was performed to alleviate symptoms maximally (Fig. 1), by removing bilateral facet joints, medial pedicular aspects, anterior compression (OPLL, herniated discs, osteophytes, etc.) and posterior cortex of the vertebrae. The techniques employed have been reported previously [7]. Altogether, five levels of $\mathrm{CD}$ were performed in four subjects.

\section{Evaluation of neurological outcomes and statistical analysis}

The follow-up included face-to-face interview, physical and imaging examinations. Neurological status was assessed using Japanese Orthopedic Association (JOA) scale for cervical myelopathy, with a maximum score of 17.0 [8]. The final neurological recovery was evaluated according to Hirabayashi's recovery rate system, with $75-100 \%$ rated as excellent, $50-74 \%$ as good, $25-49 \%$ as fair and less than $25 \%$ as unchanged or deteriorated. For those who received extra decompressions, neurological status before reoperation was recorded as the final outcomes of 


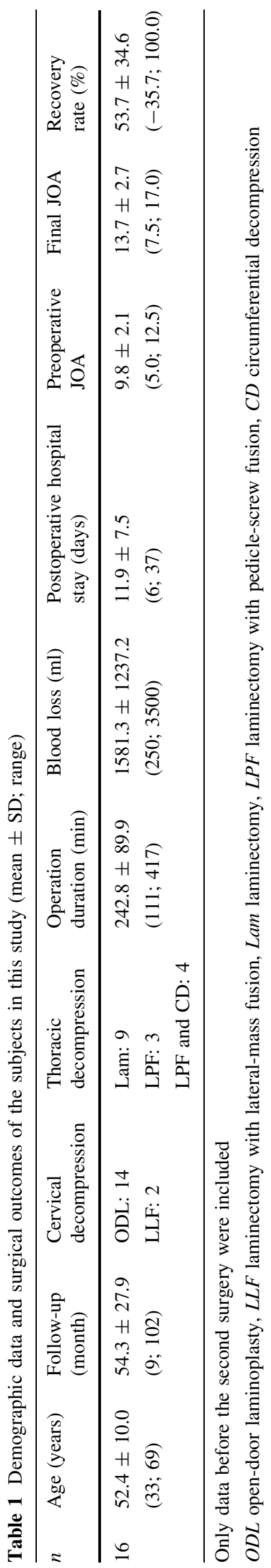

combined decompression. Clinical data were analyzed using IBM SPSS statistics 20 (Chicago, IL, USA) and presented in the form of mean \pm standard deviation (SD). Comparison analysis was performed using Fisher's exact test (two-tailed) and unpaired student $t$ test (two-tailed). Statistical significance was set at 0.05 .

\section{Results}

\section{General information}

The cohort included four males and 12 females. The average age was $52.4 \pm 10.0$ years (Table 1). Each operation lasted $242.8 \pm 89.9 \mathrm{~min}$, and the mean blood loss was $1581.3 \pm 1237.2 \mathrm{ml}$. The mean postoperative hospital stay was $11.9 \pm 7.5$ days. Eventually, each subject was followed up for $54.3 \pm 27.9$ months (Table 1).

\section{Course of neurological recovery}

After the operation, 10 subjects $(62.5 \%)$ reported instant improvement of previous symptoms, while neurological deterioration occurred in the remaining six subjects $(37.5 \%)$, among whom four suffered the worsening of muscle strength, aggravated sensory disorders in two subjects and new emerging urinary retention (UR) in two subjects. By comparison, massive blood loss, CD manipulations and cerebrospinal fluid (CSF) leakage were associated with postoperative neurological deterioration (Table 2). Before discharge, all deteriorations were successfully managed with conservative treatments (Fig. 2), including methylprednisolone, dehydrants and neurotrophic drugs, except for one subject who showed no remission of UR.

During the follow-up period, four subjects received extra thoracic decompression mainly due to the relapses of walking problems, which resulted from the remaining of anterior compression in one subject and new compression from other segments in three subjects (Fig. 3). One subject suffered the complete loss of the walking ability, and one still needed urinary catheterization. Another subject developed muscular dystrophy in the thumb-index web of right hand but admitted apparent improvement of sphincter dysfunction and leg weakness (JOA score from 9.0 to 15.0). All the latter three subjects refused extra surgical intervention.

\section{Other postoperative complications and their clinical outcomes}

Other complications included CSF leakage in 10 subjects (Table 3), lung infection in one subject, urinary infection in one subject, and pulmonary thromboembolism (PTE) in 

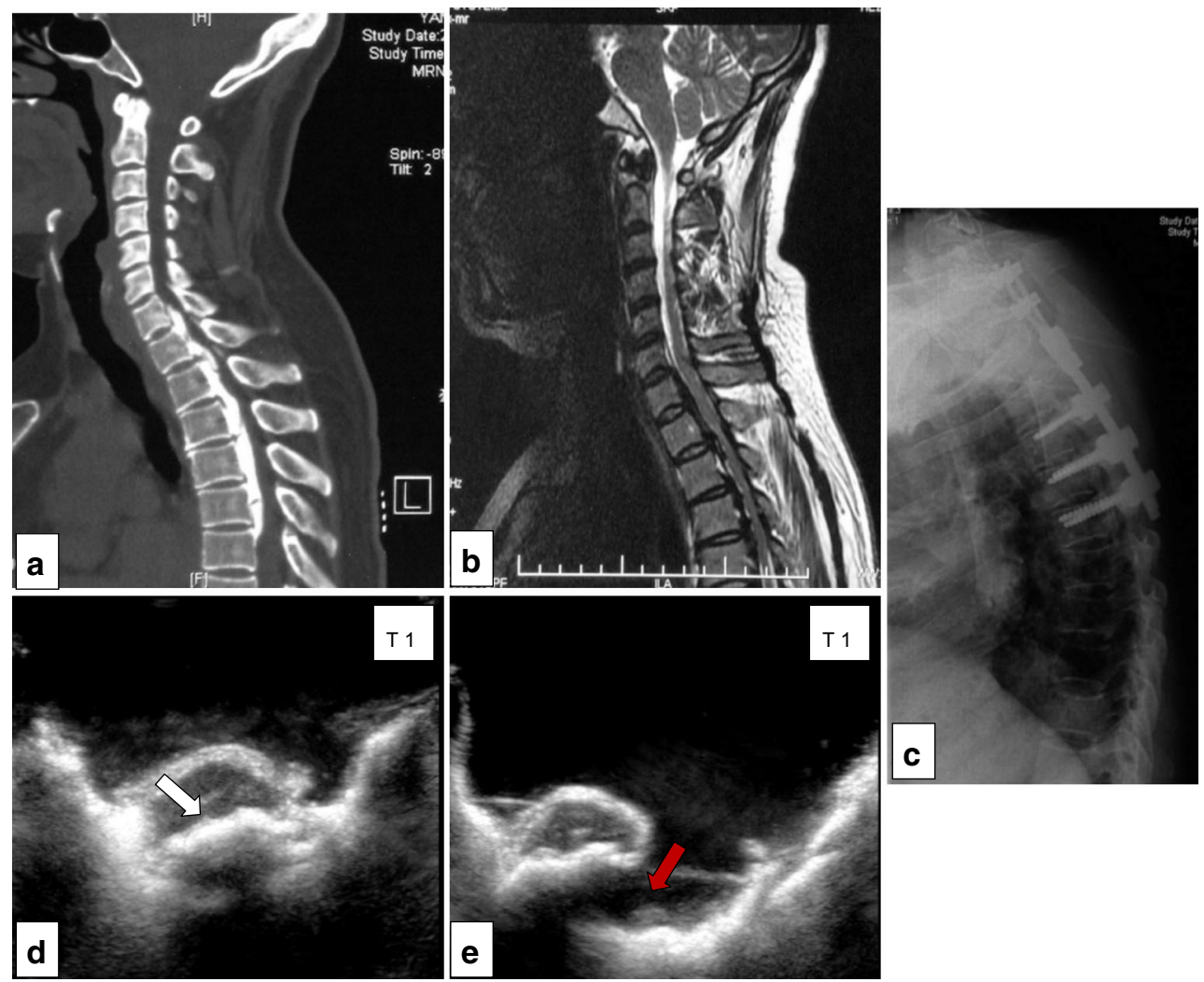

Fig. 1 Presentations of a 43-year-old female, who suffered wheelchair dependence and occasional urinary incontinence but maintained hand functions intact. Her preoperative JOA score was 11.0. a, b Displayed the continuous compression from C6 to T6 OPLL and miscellaneous signals inside the spinal cord. She underwent onestaged decompression, laminectomy with lateral-mass fusion at C6C7 and laminectomy with pedicle-screw fusion at T1-T6 (c). After laminectomy, the backward shift of spinal cord was unsatisfactory and ultrasonography revealed the persisting of severe anterior compression at $\mathrm{T} 1-\mathrm{T} 3$ (white arrow in d). Circumferential decompression was performed at these levels (red arrow in e). Instantly after the surgery, the subject displayed the complete loss of muscle strength in her right leg. She was treated with intravenous methylprednisolone and dehydrants. On the next morning, the movement of her right leg started to restore. At the final follow-up, she was still complaining of weakness in her legs and assistanceneeded when climbing stairs, but the sphincter dysfunction disappeared. Her final JOA score improved to 14.0 and thus her recovery rate was $50.0 \%$, which was good, according to Hirabayashi's system

Table 2 Comparison of factors associated with the postoperative neurological outcomes

\begin{tabular}{lllll}
\hline Items & Improved & Deteriorated & $P$ values & \\
\hline$n$ & $10(62.5 \%)$ & $6(37.5 \%)$ & Not applicable & Unpaired student $t$ test (two-tailed) \\
Age (years) & $51.5 \pm 10.9$ & $53.8 \pm 9.0$ & 0.667 & 0.236 \\
Preoperative JOA scores & $10.3 \pm 1.6$ & $8.9 \pm 2.8$ & $0.006^{*}$ & \\
Operated segments & $7.6 \pm 1.0$ & $9.5 \pm 1.4$ & $0.019^{*}$ & $0.000^{*}$ \\
Operation duration (min) & $203.8 \pm 87.2$ & $307.7 \pm 50.0$ & 0.845 & Fisher's exact test (two-tailed) \\
Blood loss (ml) & $680.0 \pm 256.3$ & $3083.3 \pm 376.4$ & 0.250 & \\
Recovery rate $(\%)$ & $52.4 \pm 25.7$ & $56.1 \pm 49.0$ & $0.008^{*}$ & \\
Thoracic OPLL & 7 & 6 & $0.034^{*}$ & \\
CD & 0 & 4 & 6 & \\
CSF leaks & 4 & & &
\end{tabular}

OPLL ossification of posterior longitudinal ligament, $C D$ circumferential decompression, CSF cerebrospinal fluid

* Statistically significant at $P<0.05$ 


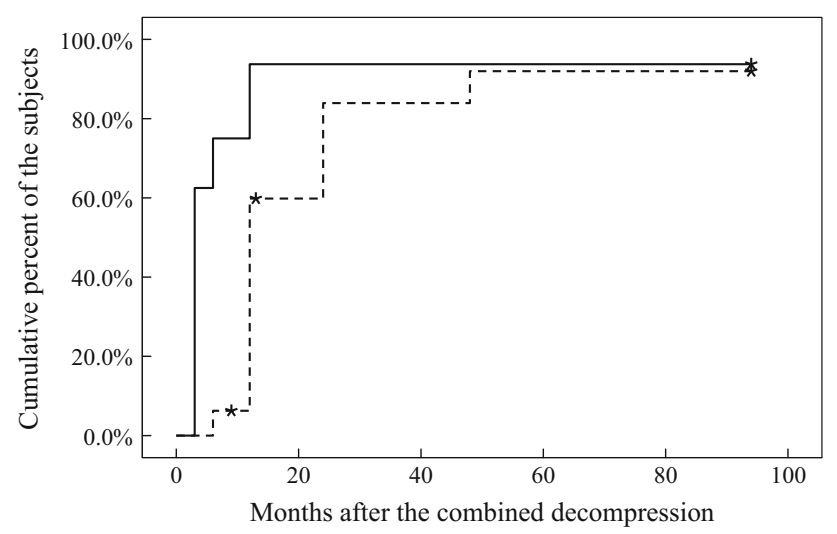

Fig. 2 Diagram of neurological recovery after the operation. Black line displayed the percent of subjects improving to a level better than preoperative neurological status. Dotted line displayed the percent of subjects reaching the peak neurological improvement during followups. Star marks represented that two subjects still showed continuing neurological improvement when having the second surgery, and that one subject did not show apparent neurological improvement until the last follow-up
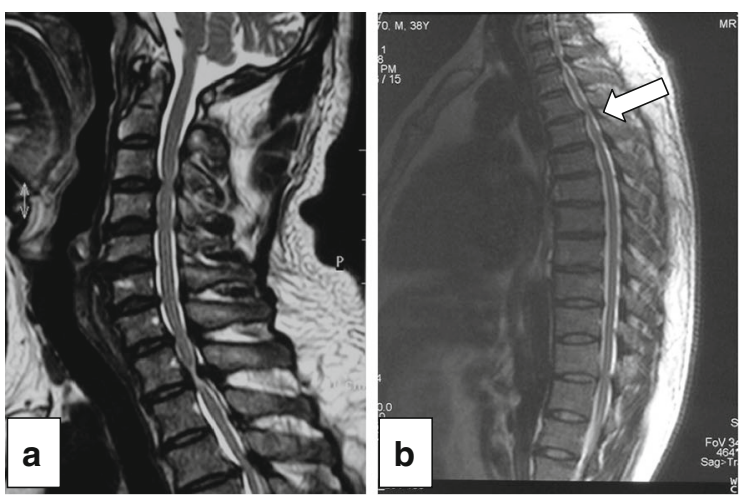

Fig. 3 Presentation of a 33-year-old male. Preoperative imaging work-up displayed multiple compression at cervical and thoracic spine (a, b), of which neurological symptoms were attributed to the compression from cervical and T1/2 OPLL at his first hospitalization. $\mathrm{He}$ underwent combined decompression at C3-T2 levels without fusion. After the surgery, his neurological improvement was significant, and JOA score peaked at 16.5. But afterwards, his neurological status started to deteriorate, and JOA score dropped to 14.0 at one subject. Three subjects developed postoperative pseudomeningoceles, which were treated with ultrasound-guided syringe aspiration to release the fluid, compressive dressing and prophylactic antibiotics. During the followup, the postoperative pseudomeningoceles resolved in two subjects but persisted in another subject, who received no extra operation but regular radiographic monitoring, due to the absence of neurologic deterioration. Besides, new sting feeling and numbness emerged in two subjects, CT scans revealed the breaking of C5 and C6 laminae from the hinging pedicles in one subject but no abnormal findings in the other subject. Since both reported significant relief of preoperative symptoms and no compression was found by CT and MRI scans, they were only prescribed with neurotrophic agents and pain killers.

\section{Evaluation of neurological recovery}

Through the combined decompression, the average JOA score was elevated from $9.8 \pm 2.1$ to $13.7 \pm 2.7$ (Table 1 ;

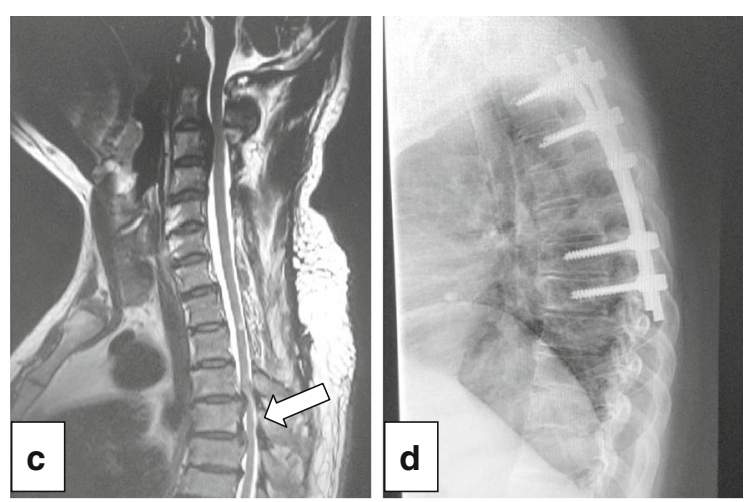

36 months after the surgery. c Taken right before the second decompression, showing the good maintenance of decompression by the combined cervico-thoracic operation but the growth and compression of OPLL at T3/4-T5/6 (arrows in b, c). Then he received laminectomy with pedicle-screw fusion at $\mathrm{T} 2-\mathrm{T} 7$ and circumferential decompression at T3/4-T4/5 for simultaneous anterior and posterior compressions (d). At the final follow-up, his JOA score improved to 17.0

Table 3 Comparison of the factors associated with cerebrospinal fluid leakage

\begin{tabular}{lllll}
\hline Items & Leakage $(n=10)$ & Non-leakage $(n=6)$ & $P$ values \\
\hline Age (year) & $54.1 \pm 9.9$ & $49.5 \pm 10.3$ & 0.391 & Unpaired student $t$ test (two-tailed) \\
Postoperative hospital stay (days) & $14.5 \pm 8.6$ & $7.5 \pm 0.8$ & 0.070 & \\
Recovery rate (\%) & $48.6 \pm 41.3$ & $62.3 \pm 19.6$ & 0.462 & Fisher's exact test (two-tailed) \\
Thoracic OPLL & 9 & 4 & 0.304 & 0.115 \\
CD & 4 & 0 & & \\
\hline
\end{tabular}

OPLL ossification of posterior longitudinal ligament, $C D$ circumferential decompression 


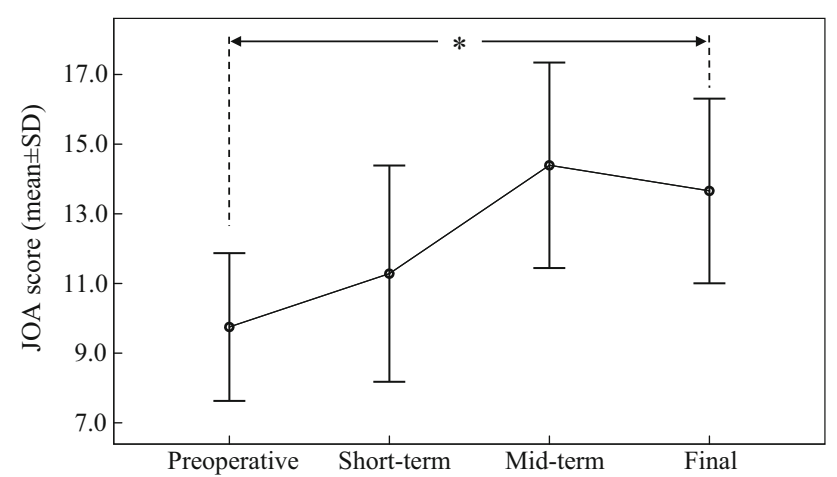

Fig. 4 JOA scores at different follow-ups. Short-term, mid-term and final follow-ups referred to around 6, 24 and 54.3 months after the operation, respectively. At the final follow-up, JOA score was significantly elevated compared with that of preoperation. *Statistical significance at $P<0.05$, unpaired student $t$ test (two-tailed)

Fig. 4). According to the Hirabayashi's classification, seven subjects were rated as excellent, four as good, two as fair, three as unchanged or deteriorated. The overall recovery rate was $53.7 \%$.

\section{Discussion}

Previous publications sporadically reported a rare clinical situation, in which symptomatic cervical and thoracic TSS simultaneously occurred $[4,5,9]$. In these reports, two surgical strategies were mentioned, namely one-staged and two-staged decompressions. However, descriptions and discussions on either approach is scarce. Therefore, more research-based evidence is necessary in order to establish a rational treatment algorithm for this pathology. In our center, 16 subjects were treated through one-staged decompression via a single posterior incision, and the main surgical manipulations were slightly different from what has been reported by Chen et al. [5].

The benefits of combined decompression are apparent, one hospitalization and one anesthesia as well as lesser medical cost, and more encouragingly, the studies on combined cervico-lumbar decompression have proven that its clinical outcomes is comparable with staged decompression $[3,6,10]$. However, it was unclear whether it is equally applicable for cervico-thoracic TSS. This study found that the postoperative courses after combined cervico-thoracic decompression were quite eventful, even though all the operations were performed by experienced surgeons. Previous publications reported that in about $30 \%$ of patients who had undergone thoracic decompression could develop postoperative neurological deterioration [7, 9-15]. It is rather alarming that $37.5 \%$ of the subjects in the present stud y suffered immediate postoperative neurological deterioration (Table 2). In fact, we were very careful on the selection of subjects and also made some modifications on surgical techniques to decrease duration of the surgery and blood loss (see "Materials and methods" section). For example, we chose ODL instead of anterior corpectomy with fusion (ACF) for cervical decompression, in order to avoid repositioning the patients and making another incision and manipulative tunnel, hoping to minimize the alteration of the spinal alignment during the operation, which could cause severe damage to the already debilitated spinal cord [14]. Urgent MRI scans were arranged for all deteriorated subjects but no remaining or new emerging compression were found. Thus, no rescue surgery was needed during the hospitalization, such as anterior decompression.

This study found that blood loss was significantly increased for the patients with postoperative neurologic deterioration (Table 2). We speculated that the main reason for massive blood loss was the prolonged operated segments in the deteriorated patients (Table 2). Besides, four of the deteriorated patients underwent $\mathrm{CD}$. This procedure was performed with an extensive removal of spinal elements; so it was often accompanied with more blood loss $[7,15]$. However, within the first year after the surgery, five deteriorated subjects improved to a level better than the preoperative neurologic status simply through conservative treatments (Fig. 2). This was an intriguing finding, implying that deterioration resulting from massive blood loss might have a more favorable prognosis than that from incomplete decompression, and this speculation well deserves a specific and comprehensive assessment by further studies. Another noticeable finding was that all of four subjects, who received thoracic decompression again 9 months to 3 years after the combined decompression, achieved postoperative neurological improvement. Though the relapse of walking inability mainly resulted from the continuing growth of thoracic ossified ligaments, as their imaging examinations revealed (Fig. 3), this phenomenon also could be interpreted as the reflection of a paradox, how to secure the sufficient decompression for all affected segments while minimizing blood loss and the area manipulating. For example, we tended to be very careful when scheming and deciding the $\mathrm{CD}$ levels for thoracic decompression, though $\mathrm{CD}$ was found to have a favorable late neurological recovery (Fig. 5).

Other unfavorable aspect of combined decompression is the high incidence of CSF leakage (62.5\%) (Table 3). Previous publications reported that OPLL was the greatest risk factor for CSF leakage and more than one third of the patients with thoracic decompression would develop this complication [7, 15-17]. Therefore, we attributed the high incidence of CSF leakage in our study to the ossified ligaments and expansive surgical areas. We adopted a set of treatment strategies for CSF leaks, including 


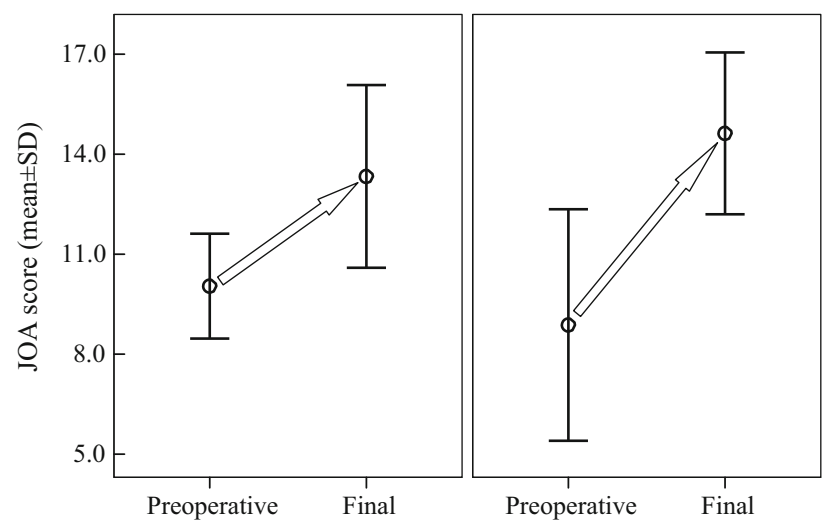

Fig. 5 Neurological recovery at the final follow-up in subjects with (right diagram) and without (left diagram) circumferential decompression $(\mathrm{CD})$ at thoracic spine. The increased mean value of JOA score was 5.8 in $\mathrm{CD}$ group, which was marginally yet nonsignificantly greater than 3.3 of non-CD group $(P=0.141$, twotailed unpaired student $t$ test)

intraoperative repair of the dural tears using fibrin glue, gelfoam and artificial dura, followed by flat bedrest and compressive dressing after the removal of drainage during the postoperative hospitalization. Though previous studies demonstrated that CSF leakage did not affect the long-term clinical outcomes $[16,18]$, some postoperative complications, including neurological deterioration, were prone to occur in the subjects with CSF leakage, for example infection and PTE. Besides, our study found that the neurological improvement predominantly occurred in the first and/or second year after this procedure (Fig. 2), and afterwards a few subjects presented with relapse of walking problems. The reoccurrence of neurological symptoms seemed earlier than reported by Chiba et al. [19] in patients with cervical myelopathy. This phenomenon, as aforementioned, was mainly related to the smaller spinal canal at the thoracic spine and more importantly, the progression of asymptomatic ossified ligaments at other thoracic segments, which was also the predominant reason for the high rate of reoperation in this study (Fig. 3).

The limits of this study were the small size of the cohort and absence of the comparison with patients who underwent two-staged decompression. Therefore, more studies on both surgical strategies are required to establish an effective surgical algorithm for cervico-thoracic TSS.

\section{Conclusion}

This study reported the clinical outcomes of one-staged combined decompression for cervical and thoracic TSS, and elaborately described the complications associated with this procedure. Though this procedure had a fair final neurological outcomes, considering the involvement of thoracic decompression in this entity, its postoperative course was frequently complicated with undesirable events, especially instant neurological deterioration and CSF leakage. Therefore, spinal surgeons should be very cautious while performing this invasive surgical procedure.

Acknowledgments We hereby thank and acknowledge the support of the Capital Foundation of Characteristic Clinical Practice and Research (Grant No. Z141107002514011) and the Foundation of Science and Research Projects for Capital Health Development (Grant No. 2014-2-4094) towards this study.

\section{Compliance with ethical standards}

Conflict of interest Funding from governmental agencies was received in support of this study.

Open Access This article is distributed under the terms of the Creative Commons Attribution 4.0 International License (http://crea tivecommons.org/licenses/by/4.0/), which permits unrestricted use, distribution, and reproduction in any medium, provided you give appropriate credit to the original author(s) and the source, provide a link to the Creative Commons license, and indicate if changes were made.

\section{References}

1. Dagi TF, Tarkington MA, Leech JJ (1987) Tandem lumbar and cervical spinal stenosis. Natural history, prognostic indices, and results after surgical decompression. J Neurosurg 66(6):842-849

2. Ghobrial GM, Oppenlander ME, Maulucci CM et al (2014) Management of asymptomatic cervical spinal stenosis in the setting of symptomatic tandem lumbar stenosis: a review. Clin Neurol Neurosurg 124:114-118

3. Kikuike K, Miyamoto K, Hosoe H et al (2009) One-staged combined cervical and lumbar decompression for patients with tandem spinal stenosis on cervical and lumbar spine: analysis of clinical outcomes with minimum 3 years follow-up. J Spinal Disord Tech 22(8):593-601

4. Park JY, Chin DK, Kim KS et al (2008) Thoracic ligament ossification in patients with cervical ossification of the posterior longitudinal ligaments: tandem ossification in the cervical and thoracic spine. Spine (Phila Pa 1976) 33(13):E407-E410

5. Chen Y, Chen DY, Wang XW et al (2012) Single-stage combined decompression for patients with tandem ossification in the cervical and thoracic spine. Arch Orthop Trauma Surg 132(9):1219-1226

6. Krishnan A, Dave BR, Kambar AK et al (2014) Coexisting lumbar and cervical stenosis (tandem spinal stenosis): an infrequent presentation. Retrospective analysis of single-stage surgery (53 cases). Eur Spine J 23(1):64-73

7. Hu P, Yu M, Liu X et al (2015) A circumferential decompressionbased surgical strategy for multilevel ossification of thoracic posterior longitudinal ligament. Spine J 15(12):2484-2492

8. Yonenobu K, Abumi K, Nagata K et al (2001) Interobserver and intraobserver reliability of the Japanese Orthopaedic Association scoring system for evaluation of cervical compression myelopathy. Spine (Phila Pa 1976) 26(17):1890-1894

9. Guo Q, Ni B, Yang J et al (2011) Simultaneous ossification of the posterior longitudinal ligament and ossification of the ligamentum flavum causing upper thoracic myelopathy in DISH: case report and literature review. Eur Spine J 20(Suppl 2):S195-S201 
10. Eskander MS, Aubin ME, Drew JM et al (2011) Is there a difference between simultaneous or staged decompression for combined cervical and lumbar stenosis? J Spinal Disord Tech 24(6):409-413

11. Onishi E, Sano H, Matsushita M (2013) Surgical treatment for thoracic myelopathy due to simultaneous ossification of the posterior longitudinal ligament and ligamentum flavum at the same level. J Spinal Disord Tech. doi:10.1097/BSD. 0000000000000059

12. Saetia K, Cho D, Lee S et al (2011) Ossification of the posterior longitudinal ligament: a review. Neurosurg Focus 30(3):E1

13. Kawahara N, Tomita K, Murakami H et al (2008) Circumspinal decompression with dekyphosis stabilization for thoracic myelopathy due to ossification of the posterior longitudinal ligament. Spine (Phila Pa 1976) 33(1):39-46

14. Yamazaki M, Mochizuki M, Ikeda Y et al (2006) Clinical results of surgery for thoracic myelopathy caused by ossification of the posterior longitudinal ligament: operative indication of posterior decompression with instrumented fusion. Spine (Phila Pa 1976) 31(13):1452-1460
15. Takahata M, Ito M, Abumi K et al (2008) Clinical results and complications of circumferential spinal cord decompression through a single posterior approach for thoracic myelopathy caused by ossification of posterior longitudinal ligament. Spine (Phila Pa 1976) 33(15):1199-1208

16. Hannallah D, Lee J, Khan M et al (2008) Cerebrospinal fluid leaks following cervical spine surgery. J Bone Joint Surg Am 90(5):1101-1105

17. Li M, Meng H, Du J et al (2012) Management of thoracic myelopathy caused by ossification of the posterior longitudinal ligament combined with ossification of the ligamentum flavuma retrospective study. Spine J 12(12):1093-1102

18. Syre P, Bohman LE, Bltuch G (2014) Cerebrospinal fluid leaks and their management after anterior cervical discetomy and fusion: a report of 13 cases and a review of the literature. Spine (Phila Pa 1976) 39(16):E936-E943

19. Chiba K, Ogawa Y, Ishii K et al (2006) Long-term results of expansive open-door laminoplasty for cervical myelopathy-average 14-year follow-up study. Spine (Phila Pa 1976) 31(26):2998-3005 